\title{
Tradução, adaptação transcultural e avaliação psicométrica da versão em português (brasileiro) do 14-item Health Literacy Scale
}

\author{
Translation, cross-cultural adaptation and psychometric evaluation \\ of Brazilian Portuguese version of the 14-item Health Literacy Scale
}

Marília Jesus Batista (https://orcid.org/0000-0002-0379-3742) ${ }^{1}$

Ana Carolina de Paula Marques (https://orcid.org/0000-0001-6866-4901) ${ }^{1}$ Manoelito Ferreira Silva Junior (https://orcid.org/0000-0001-8837-5912) ${ }^{2}$

Gizelton Pereira Alencar (https://orcid.org/0000-0002-2354-9050) ${ }^{3}$

Maria da Luz Rosário de Sousa (https://orcid.org/0000-0002-0346-5060) ${ }^{1}$
${ }^{1}$ Departamento de Ciências da Saúde e Odontologia Infantil, Faculdade de Odontologia de Piracicaba, Universidade Estadual de Campinas. Av. Limeira 901, Bairro Areião. 13414-903 Piracicaba SP Brasil. mariliajbatista@yahoo. com.br

${ }^{2}$ Departamento de Odontologia, Universidade Estadual de Ponta Grossa. Ponta Grossa PR Brasil.

${ }^{3}$ Departamento de Epidemiologia, Faculdade de Saúde Pública, Universidade de São Paulo. São Paulo SP Brasil.

\begin{abstract}
We evaluated the psychometric properties of the Health Literacy Scale - 14 (HLS-14), the Brazilian Portuguese version. In the methodological study with a cross-sectional design, the following were performed: translation, cross-cultural adaptation and evaluation of the psychometric properties. After being subjected to a committee of experts, translated and adapted, the instrument was pre-tested with 52 adults and applied to 143 adults and older people of Piracicaba-SP. Internal consistency was assessed based on the Kendall correlation coefficient and Cronbach's alpha ( $>0.70)$, and the confirmatory factor analysis (CFA) was conducted using the chi-square test, the Standardized Residual Mean Square Root (SRMR), the Root Mean Square Error of Approximation (RMSEA) (<0.05), the Comparative Fit Index (CFI) (>0.95) and the Tucker-Lewis Index (TLI) (>0.95). The analysis of operational equivalence showed agreement between most of the questions, exception questions 6, 8, 9 and 14. Cronbach's alpha was 0.82 . There was reasonable adjustment in the CFA: $C F I=0.886, T L I=0.86, R M S E A=0.085$ (90\%CI: 0.065-0.105), SRMR $=0.071$, chi-square (74 degrees of freedom $)=149.510, p<0.001$. The exclusion of question 5 increased the adjustment level to satisfactory. The Brazilian Portuguese version of HLS-14 can be considered a valid health literacy assessment instrument.
\end{abstract}

Key words Validation Studies, Health Literacy, Epidemiologic Measurements
Resumo Foram avaliadas as propriedades psicométricas da versão brasileira do 14-item Health Literacy Scale (HLS-14). No estudo metodológico com delineamento transversal realizou-se tradução, adaptação transcultural e avaliação das propriedades psicométricas. Depois de passar por comitê de especialistas, traduzido e adaptado, o instrumento foi pré-testado em 52 adultos, e aplicado em 143 adultos e idosos de Piracicaba-SP. A consistência interna foi avaliada através do coeficiente de correlação de Kendall e a de Cronbach $(>0,70)$ e a análise fatorial confirmatória (AFC) por meio do teste de qui-quadrado, raiz quadrada média residual padronizada (SRMR), raiz da média dos quadrados dos erros de aproximação (RM$S E A)(<0,05)$, indice de ajuste comparativo (CFI) $(>0,95)$ e indice de Tucker-Lewis (TLI) $(>0,95)$. A equivalência operacional apresentou concordância entre as questões, com exceção das questões 6, 8, 9 e 14. O coeficiente $\alpha$ de Cronbach foi 0,82 . Houve ajuste razoável na $A F C, C F I=0,886$, $T L I=0,86$, RMSEA =0,085 (IC90\%: 0,065-0,105), $S R M R=0,071$, qui-quadrado (74 graus de liberdade) $=149,510, p<0,001$. A exclusão da questão 5 elevou os indices de ajuste a niveis satisfatórios. A versão brasileira do HLS-14 foi considerada válida para mensurar literacia em saúde.

Palavras-chave Estudos de validação, Alfabetização em saúde, Medidas em epidemiologia 


\section{Introdução}

A literacia em saúde pode ser definida como o conjunto de aptidões sociais e cognitivas que determinam a motivação e a capacidade dos indivíduos em ter acesso, compreender e utilizar informações para promover e manter boa saúde $\mathrm{d}^{1,2}$. Associada à autonomia no cuidado em saúde, a literacia em saúde tem sido relacionada ao empoderamento $^{3}$ e por isso, torna-se uma importante estratégia de promoção de saúde ${ }^{4}$. Assim, estudos que avaliem a mensuração da literacia em saúde são de grande relevância, e um desafio no que tange a complexidade de suas dimensões.

Estudos mostram que indivíduos com maior literacia em saúde estão propensos a adotar comportamentos mais saudáveis ${ }^{5-7}$, por serem capazes de compreender as orientações que lhe foram feitas como prescrição, marcação de consulta, rótulos de medicamentos e outras instruções para o autocuidado ${ }^{8}$. No entanto, o baixo nível de literacia em saúde está associado com maiores taxas de hospitalização, uso de serviços de emergência, erros na ingestão de medicamentos com relação à dosagem, menor conhecimento sobre autocuidado em saúde e maior risco de mortalidade. Tem sido evidenciado na literatura que os indivíduos com baixa literacia buscam menos cuidados preventivos e possuem manejo menos adequado de doenças crônicas ${ }^{5,7,9-11}$.

A literacia em saúde pode ser abordada em diferentes dimensões, sendo funcional, comunicativa e crítica. A literacia funcional consiste em possuir habilidades básicas de leitura e escrita para situações do cotidiano, como ter domínio de informações sobre riscos de saúde e a utilização de serviços de saúde. A literacia comunicativa representa habilidades cognitivas mais avançadas juntamente com habilidades sociais que possibilitam as pessoas e as comunidades a agirem de modo independente. Trata-se da habilidade de extrair informações de diferentes meios de comunicação e aplicar a nova informação para situações pessoais, promovendo mudança nas circunstâncias. A literacia crítica avalia a habilidade de analisar criticamente as informações disponíveis, e usar essas informações para exercer maior controle sobre os eventos da vida e situações, e trata-se do processo de empoderamento dos indivíduos ${ }^{4}$.

Embora haja uma diversidade de instrumentos na literatura, a maioria avalia apenas a literacia no nível funcional, também conhecida como letramento ou alfabetização em saúde, com enfoque predominante na leitura e escrita ${ }^{12}$, como é o caso dos Rapid Estimate of Adult Literacy in Medicine (REALM) ${ }^{13}$ e sua versão reduzida, o REAL$\mathrm{M}-\mathrm{R}^{14}$, Test of Functional Health Literacy in Adults (TOFHLA) ${ }^{7}$ e sua versão curta (S-TOFHLA) ${ }^{15} \mathrm{e}$ o Rapid Estimate of Adult Literacy in Dentistry (REALD-30) ${ }^{16}$, dentre outros.

Um dos poucos instrumentos relacionados à literacia em saúde que mensura as dimensões crítica e comunicativa, foi desenvolvido por Ishikawa et al. ${ }^{6}$ e posteriormente foi modificado e ampliado por Suka et al. ${ }^{17}$, e denominado de 14 item Health Literacy Scale (HLS-14). O HLS-14 é o único questionário que avalia os três níveis da literacia: funcional, comunicativa e crítica e a versão original foi validado em adultos japoneses ${ }^{17} \mathrm{e}$ apresenta uma versão validada na Holanda ${ }^{18}$.

Tendo em vista a grande relevância do uso de um instrumento capaz de avaliar a literacia em saúde em seus três níveis, o objetivo do estudo foi avaliar as propriedades pscométricas da versão em português (brasileiro) do 14-item Health Literacy Scale em adultos e idosos brasileiros.

\section{Metodologia}

\section{Desenho do estudo e local da pesquisa}

Trata-se de um estudo metodológico com delineamento transversal, em que foram realizados a tradução, adaptação transcultural e avaliação das propriedades psicométricas do 14-item Health Literacy Scale (HLS-14) realizado na cidade de Piracicaba, São Paulo, Brasil.

\section{Aspectos Éticos}

O processo para avaliação das propriedades psicométricas do instrumento HLS-14 para o português (brasileiro) foi previamente autorizado pelos autores do instrumento ${ }^{17}$. Esta pesquisa foi aprovada pelo Comitê de Ética em Pesquisa da Faculdade de Odontologia de Piracicaba (CEP-FOP/UNICAMP).

\section{Instrumento}

O Health Literacy Scale-14 (HLS-14) criado e validado por Suka et al. ${ }^{17}$ contém quatorze questões em escala likert de cinco pontos e avalia três dimensões da literacia: funcional (LF) e comunicativa (LCo) com 5 itens cada, e crítica (LCr) com 4 itens.

$\mathrm{O}$ processo de adaptação transcultural e avaliação das propriedades psicométricas ${ }^{19-21}$ 
considerou 4 etapas: equivalência conceitual, equivalência de itens e semântica, equivalência operacional e equivalência de mensuração.

\section{Equivalência conceitual}

Nessa etapa estudou-se a concepção e o processo de equivalência conceitual do instrumento original 14-item Health Literacy Scale (HLS-14), a partir do instrumento de Ishikawa et al. ${ }^{6}$ que foi aplicado no baseline do estudo ${ }^{6}$. O questionário foi medido em escala likert de 5 pontos, com opções de respostas que variavam de concordo totalmente a discordo totalmente. Vale ressaltar que as cinco primeiras questões, relacionadas à literacia funcional, apresenta um escore de pontos invertida, uma vez que concordar com as afirmativas está relacionado a uma baixa literacia, enquanto para as demais nove questões, relacionadas a literacia comunicativa e crítica concordar com a assertiva está relacionado a uma alta literacia em saúde. As pontuações dos itens eram somadas para cada voluntário e obtidas então a pontuação final, que indica o nível de literacia em saúde. Quanto maior o escore, maior a literacia em saúde ${ }^{17}$.

\section{Equivalência de itens e semântica}

Para equivalência de itens e semântica, reuniões entre os quatro pesquisadores, que formaram o comitê de especialistas desta etapa, ocorreram para discutir os conceitos do construto original HLS-14 e a relevância para sua aplicação em uma população brasileira. O comitê de especialistas foi composto por quatro pesquisadores, sendo dois com fluência na língua inglesa.

Para a equivalência semântica o questionário original foi traduzido da língua inglesa de forma literal para o Português Brasileiro. Essa etapa foi realizada por dois tradutores com proficiência em ambos os idiomas, inglês e português, um de língua materna inglesa com proficiência em português brasileiro e outro da língua materna portuguesa (brasileira) com proficiência em inglês, ambos não cientes dos objetivos da pesquisa.

Houve um consenso entre os quatro pesquisadores após essa etapa, sobre a tradução de cada vocábulo, para realização da equivalência de itens e semântica. Os pesquisadores concluíram que seriam necessárias reformulações e adaptações de alguns termos para melhor compreensão do questionário. Após modificações, os autores enviaram o questionário retrotraduzido ao primeiro autor (e autor de correspondência) do estudo original que validou o questionário no Japão, para adequada análise e emissão de parecer. Posteriormente a análise semântica das questões do instrumento HLS-14, obteve-se uma versão traduzida e adaptada ao Português Brasileiro.

\section{Equivalência operacional}

O questionário foi aplicado em uma amostra inicial de 52 voluntários da Unidade de Saúde Boa Esperança I, da cidade de Piracicaba-SP selecionados aleatoriamente, para testar o questionário adaptado e verificação da compreensão das perguntas e respostas. Os voluntários relataram sobre a compreensão do instrumento. $\mathrm{O}$ instrumento foi reaplicado nos mesmos indivíduos e foi realizado Teste de Correlação de Kendall entre os dois momentos para avaliar confiabilidade da compreensão.

Para garantir a manutenção da adaptação cultural e conceitual entre a versão original e adaptada, a versão final foi retrotraduzida por outro tradutor independente de língua materna inglesa, com proficiência em ambas as línguas para verificar se manteve o sentido do idioma original.

\section{Equivalência de mensuração}

Para esta etapa o questionário traduzido e adaptado foi aplicado em uma população de adultos e idosos que faz parte de um estudo de coorte prospectivo no município de Piracicaba, localizado no interior de São Paulo.

A amostra desta etapa foi obtida durante o estudo "Coorte de Saúde Bucal de Adultos de Piracicaba-SP"22, iniciado em 2011 (baseline) com uma amostra representativa de adultos entre 20 e 64 anos residentes do município de Piracicaba-SP, estratificada por diferenças existentes nas condições de saúde bucal nos diferentes grupos etários: adultos jovens (20 a 44 anos) e adultos mais velhos (45 a 64 anos), utilizando dois cálculos. Adotou-se o efeito de desenho (deff) de 1,5, margem de erro de $10 \%$, intervalo de confiança de $95 \%$, e dados de prevalência da experiência de cárie para cada grupo etário (70,2\% e 90,9\% respectivamente).

A população de Piracicaba em 2000 era 368.836 no total, sendo que 202.131 indivíduos encontravam-se entre 20 e 64 anos. O tamanho amostral para adultos de 20 a 44 foi 172 e para adultos de 45 a 64 anos foi 68, estimando 240 indivíduos. Foram considerados para o sorteio 11 domicílios para cada um dos 30 setores cen- 
sitários. A seleção amostral foi planejada a partir de dados do censo de 2000 fornecidos pelo IBGE, que eram os dados compilados mais recentes no momento do estudo. A média de moradores na faixa etária por domicílio no censo de 2000 foi 2,49 e a média de domicílios por setor foi 177,75. Foram sorteados, segundo a técnica de amostragem probabilística, 30 setores censitários, mais 2 para serem suplentes. Em cada setor, foram sorteadas 11 residências, segundo a fração que variou determinada pelo número de domicílios. Foi examinado um adulto por domicílio e totalizaram 248 participantes no baseline.

Em 2015, houve a realização do acompanhamento (follow-up), e houve a aplicação do HLS14, para avaliação das propriedades psicométricas. Nesse momento, o critério de inclusão do estudo foram indivíduos participantes do estudo em 2011 em residência selecionada no município de Piracicaba-SP por amostragem probabilística que possuíam idade entre 20-64 anos em 2011. A amostra no estudo longitudinal em 2015 foi de 143 adultos entre 23-69 anos ${ }^{22}$.

A coleta de dados desta etapa ocorreu nos domicílios entre os meses de junho a setembro de 2015. Foi realizado o exame clínico bucal, uma entrevista e a aplicação do instrumento HLS-14. A aplicação do instrumento teve duração aproximada de 15 minutos. Além desse questionário, cada voluntário respondeu a outras informações sobre fatores demográficos e socioeconômicos.

\section{Análise estatística}

Para a análise estatística utilizou-se o Statistical Package for the Social Sciences (SPSS), versão 20.0 e Excel $^{\circ}$. Foram calculados o coeficiente de correlação de Kendall e o teste $\alpha$ de Cronbach, sendo o instrumento considerado adequado quando a consistência interna é maior ou igual à $0,70^{23}$. Para a avaliação da validade de estrutura fatorial foi utilizada a análise fatorial confirmatória utilizando o software Mplus, a partir da estrutura apresentada na proposta do modelo por Suka et al. ${ }^{17}$. Considerou-se a estimação por máxima verossimilhança robusta dado que as variáveis categóricas foram medidas em escala Likert de 4 ou mais ${ }^{24}$. $\mathrm{O}$ ajuste do modelo foi verificado por meio do teste de qui-quadrado e dos índices: a raiz quadrada média residual padronizada (SRMR) ponto de corte adotado foi $<0,80^{25}$, a raiz da média dos quadrados dos erros de aproximação (RMSEA), o índice de ajuste comparativo (CFI) e o índice de Tucker-Lewis (TLI). Em geral, RMSEA $<0,05$ indica um bom ajuste, mas são razoáveis valores entre 0,05 e 0,08 . Os valores de CFI e TLI $>0,95$ indicam um bom ajuste ${ }^{25}$.

A validade convergente dos fatores foi obtida com as medidas de Variância Extraída Média (VEM) e Confiabilidade Composta (CC). Foram considerados adequados valores de VEM $\geq 0,50 \mathrm{e}$ CC $\geq 0,70$. Para avaliar a validade discriminante compararam-se as VEM de cada fator com o quadrado da correlação entre os fatores $\left(\rho_{\mathrm{ij}}{ }^{2}\right)$. Considera-se que há validade discriminante quando os valores de $\operatorname{VEM}_{\mathrm{i}}$ e VEM $\mathrm{j} \geq\left(\rho_{\mathrm{ij}}{ }^{2}\right)^{26}$. Para avaliar possíveis efeitos de teto/chão foi calculada a soma e avaliada sua distribuição, além de identificar diferentes distribuições de sexo e idade nas respostas dos itens, utilizando a análise de variância para comparar médias e o teste de Levene para comparar variâncias da soma dos itens.

\section{Resultados}

O questionário foi traduzido por dois tradutores, e após esta etapa foi realizada avaliação pelo comitê de especialistas para um consenso entre os quatro pesquisadores envolvidos. Após avaliação dos pesquisadores do presente estudo, o questionário retrotraduzido e adaptado foi enviado ao autor do questionário original que sugeriu modificações nas questões 10, 12 e 14 . Ajustes para evitar possíveis dificuldades na compreensão de algumas das questões foram efetuados na linguagem das questões, como apresentado no Quadro 1.

No enunciado que antecede o fator da literacia comunicativa houve necessidade de ajustes para melhorar a compreensão. A frase foi reescrita para "Quando você lê bulas de remédio, responda". Na questão 1 , foi realizada a substituição do termo Caracteres por palavras, pois poderia expressar melhor o sentido no Português Brasileiro. Na questão 6, houve a substituição dos termos "diversas fontes" por "em vários lugares". Assim como na questão 7, que foi permutado o vocábulo "extraio" por "encontro" não alterando o sentido da questão. Sequencialmente, a questão 8 , houve a troca da palavra "obtive" por "encontrada". Enquanto que na questão de número 9 foi permutado o vocábulo "informo" por "falo". Na questão de número 10 , houve alteração da palavra "aplico" pelo termo "coloco em prática", e ainda, o vocábulo "obtive" por "encontrada". Da mesma forma que na décima segunda questão, foi permutado o termo "levo em conta" pela palavra "sei". Semelhantemente, na questão 14, houve mudança na expressão, que inicialmente era: "Eu junto informações em que posso basear minhas informações sobre saúde" após as 
Quadro 1. Instrumento HLS-14 em sua versão original, traduzida e após adaptação transcultural. Piracicaba-SP, Brasil, 2015.

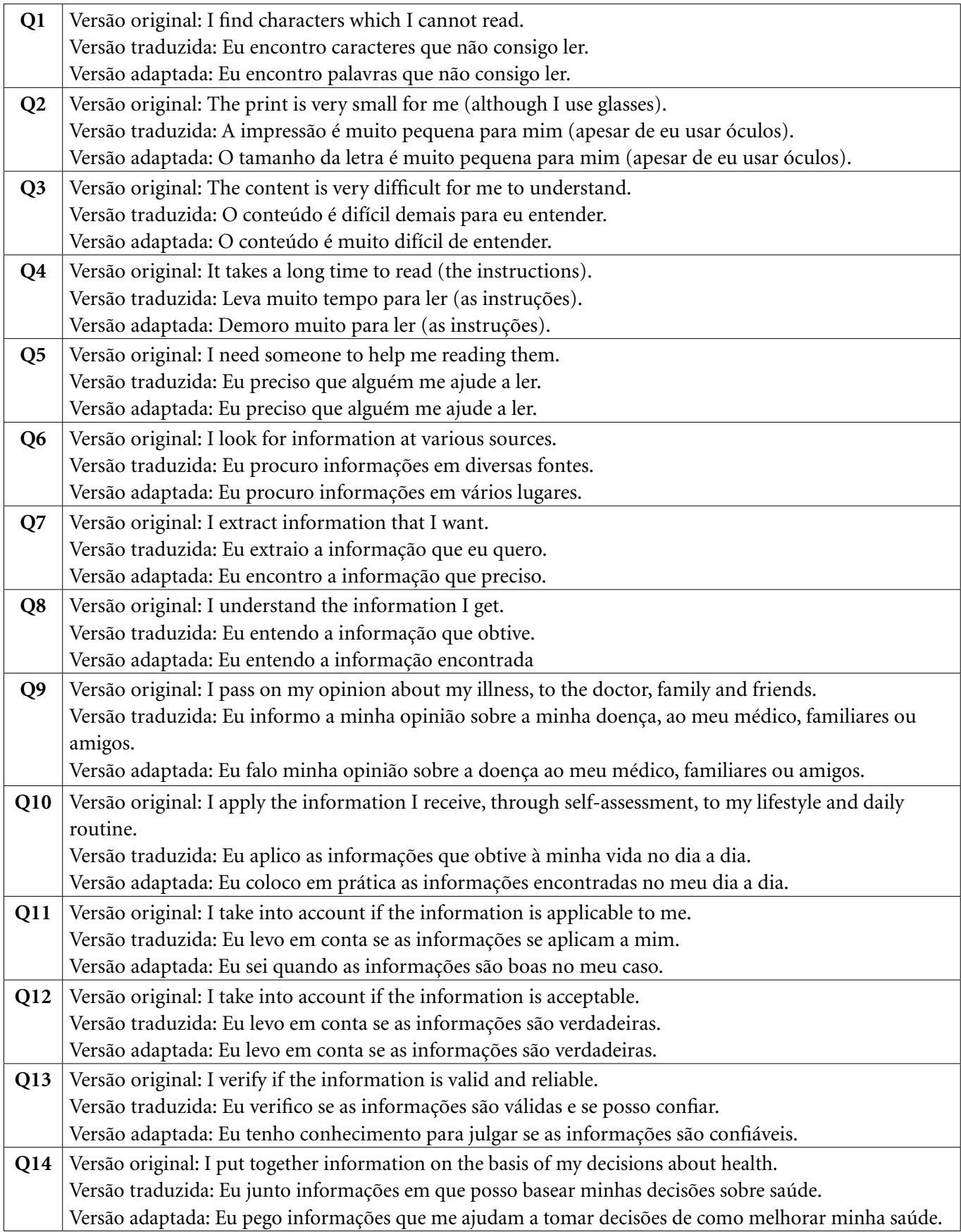

adaptações, assumiu a seguinte forma: "Eu pego informações gerais que me ajudam a tomar decisões de como melhorar minha saúde".

Segundo o relato geral dos voluntários, o instrumento foi considerado importante para avaliar a sua capacidade em entender as instruções dadas pelos profissionais de saúde.
A versão final do instrumento foi aplicada em um pré-teste com 52 usuários de unidades de saúde. Após o teste de correlação de Kendall todas as questões foram associadas, quando comparadas com a primeira e segunda aplicação do questionário, com exceção das questões $6,8,9$ e $14(\mathrm{p}<0,05)$. 
Quando o questionário de literacia foi aplicado em uma amostra de adultos e idosos de Piracicaba $(n=143)$, a maioria dos examinados foram mulheres, entre 49-69 anos, com mais de 11 anos de estudo (Tabela 1). Houve uma consistência interna do instrumento calculada através do coeficiente $\alpha$ de Cronbach de 0,82. Para cada dimensão houve um valor do coeficiente $\alpha$ de Cronbach. Para a literacia funcional $\alpha=0,66$, literacia comunicativa $\alpha=0,90$ e literacia crítica $\alpha=0,81$.

Aplicando a análise fatorial confirmatória considerando o modelo de Suka et al. ${ }^{17}$ este apresentou ajuste razoável de todos os índices: $\mathrm{CFI}=$ 0,886, TLI $=0,86$, RMSEA = 0,085 (IC90\%: 0,065$0,105)$, SRMR $=0,071$, qui-quadrado $(74$ graus de liberdade $)=149,510, p<0,001$. Observou-se correlação alta $(0,80)$ entre LCom e LCr. A exclusão da questão 5 na análise do instrumento elevou os índices de ajuste para níveis satisfatórios: CFI $=0,930$, TLI $=0,912$, RMSEA $=0,075 \quad($ IC90\%: 0,046-0,093), SRMR $=0,064$, qui-quadrado $(64$ graus de liberdade $)=104,288, p<0,001$. Portanto, os critérios usuais da AFC foram atendidos. $\mathrm{O}$ modelo conta com $26 \%$ da variância de LF, $74 \%$ em LCo, e $53 \%$ em LCr. As estimativas padronizadas, as correlações entre os fatores, desvio-padrão dos erros estão na Figura 1.

Segundo os valores de Variância Estimada Média (VEM) e CC obtidos, os fatores F2 (LCom) e F3 (LCr) apresentaram validade convergente e discriminante, entretanto o fator F1 (LF) apresentou problemas de validade discriminante (Tabela 2).

A soma dos itens varia entre 14 a 62 , com percentis 25,50 e $75(42,46,50)$. O valor de assimetria $(-0,86)$ indicou não haver muita simetria, e curtose $(4,53)$ sugeriu que as caudas são mais pesadas. Não houve diferenças nas distribuições dos itens segundo sexo (Anova: $p=0,5064$, Levene: $p=0,6912$ ). Para a idade, não há diferenças nas variâncias dos itens segundo quartis de idades ( $p=0,1773)$, no entanto, houve diferença de médias $(p=0,004)$ : até 32 anos os escores tenderam a serem maiores (média $=48,58$ ) e decrescem segundo a idade, entre 33-45 anos (média $=46,44)$, entre 46 e 54 anos (média $=44,17)$ e acima de 55 anos (média $=42,38$ ).

A média do score total HLS-14 foi de 45,16, e o desvio padrão (DP) de 7,8 nesta amostra de adultos. Um total de $53,8 \%(\mathrm{n}=77)$ desta amostra apresenta alta literacia. Pode ser observado que $43,4 \%(n=62)$ dos entrevistados relataram dificuldade em relação à compreensão de palavras contidas em bulas de remédio, 46,2\% ( $\mathrm{n}=$
Tabela 1. Características demográficas e socioeconômicas dos adultos e idosos residentes em Piracicaba-SP, Brasil, 2015.

\begin{tabular}{lcc}
\hline \multicolumn{1}{c}{ Variáveis } & n & $\%$ \\
\hline Renda familiar & & \\
Alta $(\geq 4 \mathrm{SM})$ & 48 & 33,6 \\
Média $(2-3 \mathrm{SM})$ & 33 & 23,1 \\
$\quad$ Baixa $(\leq 1 \mathrm{SM})$ & 47 & 32,9 \\
Sexo & & \\
$\quad$ Feminino & 40 & 28,0 \\
$\quad$ Masculino & 103 & 72,0 \\
Idade & & \\
23 a 48 anos & 68 & 47,6 \\
$\quad 49$ a 69 anos & 75 & 52,4 \\
Escolaridade (anos) & & \\
11 ou mais & 78 & 54,5 \\
5 a 10 & 34 & 23,8 \\
Até 4 & 31 & 21,7 \\
\hline
\end{tabular}

66) consideram a impressão das letras de bulas de medicamentos pequenas e $60,1 \%(n=86)$ dos voluntários, afirmam que não buscam informações adicionais que os ajudam a tomar melhores decisões sobre sua saúde (Tabela 3).

\section{Discussão}

É de extrema importância o conhecimento da literacia em saúde de uma população, pois tem sido apontada como estratégia de promoção de saúde para a $\mathrm{OMS}^{1}$. Mensurar a literacia em saúde tem sido um desafio e traz um avanço para a Saúde Pública no Brasil, que recentemente vem desenvolvendo estudos neste campo. No Brasil, a maioria das avaliações das propriedades psicométricas de instrumentos de literacia em saúde trazem resultados de compreensão e capacidade de leitura, enquanto o presente estudo avaliou um instrumento que propõe mensurar a literacia em saúde em seus três níveis: funcional, comunicativa e crítica. A literacia em saúde deve ser entendida como um aspecto que extrapola a capacidade de leitura para questões sociais e políticas que implicam no exercício da cidadania ${ }^{27}$. O presente estudo demonstra que a versão em português (brasileiro) do instrumento HLS-14 demonstrou ser válida, embora sejam necessários alguns ajustes para avaliar a literacia em saúde na população adulta brasileira estudada de forma mais adequada. A aplicação do instrumento é simples e rápida, cerca 


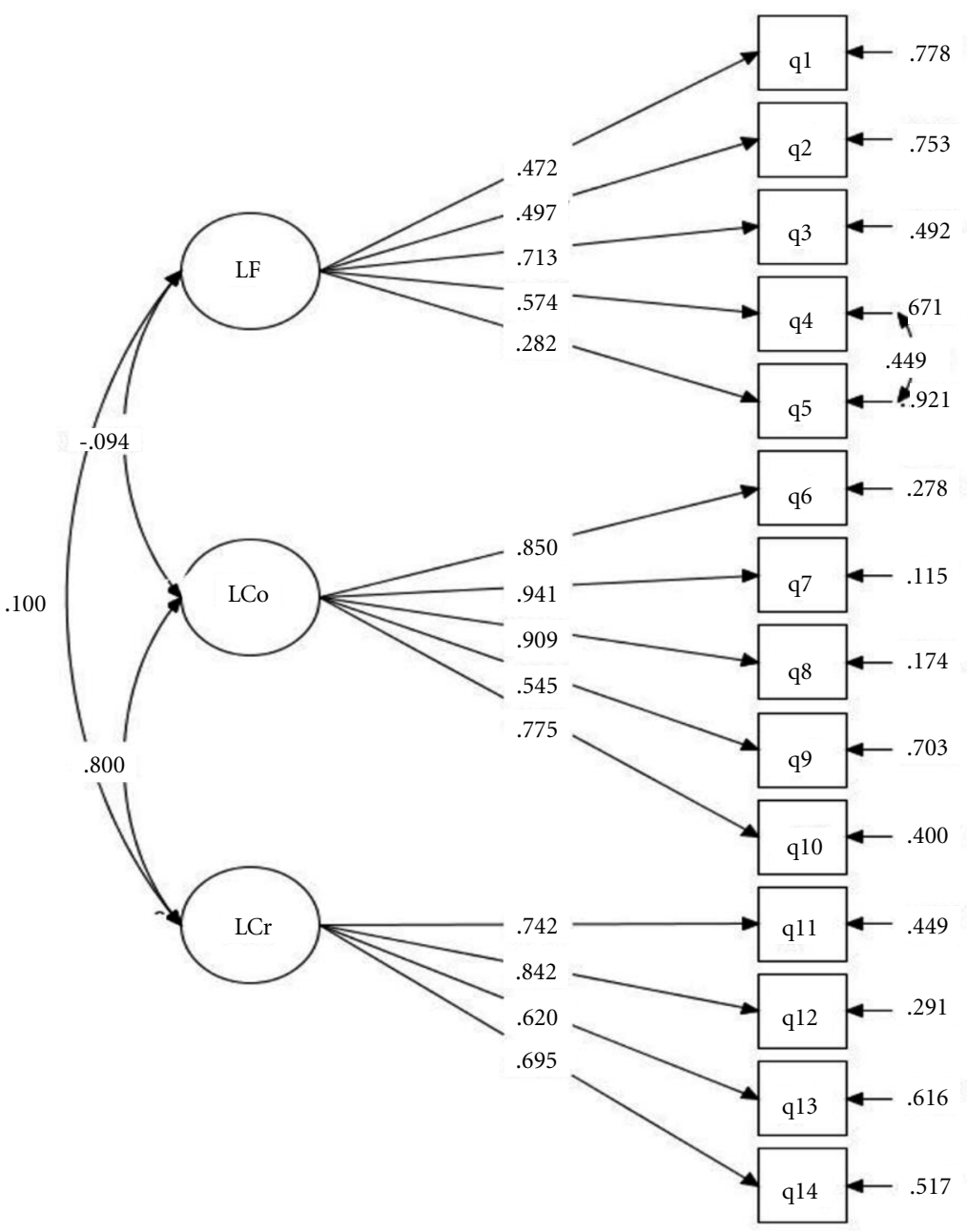

Figura 1. Análise fatorial confirmatória do modelo de literacia em saúde.

LF - Literacia Funcional; LCo - Literacia Comunicativa; LCr - Literacia Crítica; q - Questão (1 a 14).

de quinze minutos, sendo viável para ser utilizado em ambientes clínicos ou em estratégias coletivas.

Os autores do estudo de validação do instrumento HLS-14 para língua holandesa ${ }^{18}$ apontaram uma falta de instrumentos que sejam capazes de avaliar a literacia nos seus três níveis e com aplicabilidade em diferentes populações. Este fato impactou na escolha deste instrumento no presente estudo, pois apresenta abrangência nas três dimensões de literacia em saúde para verificar sua aplicabilidade na população brasileira.
O instrumento mostrou-se aplicável, no presente estudo, após a realização de adaptações na semântica, que melhoraram a compreensão das questões. No presente estudo, houve discordância na equivalência operacional em quatro questões do questionário (questões 6, 8, 9 e 14), referentes às dimensões comunicativa e crítica. Esse fato também foi apontado no estudo holandes ${ }^{18}$, onde as questões 6, 9 e 14 também apresentaram problemas de compreensão de significado, demonstrando que mesmo em duas culturas diferentes 
Tabela 2. Valores de Variância Estimada Média (VEM) e Confiabilidade Composta (CC).

\begin{tabular}{lccrccc}
\hline Modelo & VEM & CC & $\begin{array}{c}\text { Raiz } \\
\text { (VEM) }\end{array}$ & $\begin{array}{c}\text { F1 } \\
\text { (LF) }\end{array}$ & $\begin{array}{c}\text { F2 } \\
\text { (Lcom) }\end{array}$ & $\begin{array}{c}\text { F3 } \\
\text { (LCr) }\end{array}$ \\
\hline F1 & 0.525 & 0.813 & 0.725 & 1.000 & & \\
F2 & 0.664 & 0.927 & 0.815 & 0.002 & 1.000 & \\
F3 & 0.668 & 0.829 & 0.817 & 0.020 & 0.561 & 1.000 \\
\hline
\end{tabular}

LF: Literacia Funcional; LCom: Literacia Comunicativa; LCr Literacia Crítica.

as dificuldades foram semelhantes. Dessa forma, estudos futuros sobre o instrumento devem rever a semântica destas questões mencionadas.

A versão em Português Brasileiro do instrumento HLS-14 demonstrou, no presente estudo, uma boa consistência interna, pois segundo $\mathrm{Kline}^{23}$, valores de alfa de Cronbach maiores que 0,70 são considerados adequados entre instrumentos destinados à aplicação de testes de habilidade. Em estudos com amostra heterogênea são esperados coeficientes de confiabilidade mais elevados, principalmente em grupos populacionais, por exemplo, residentes de uma cidade ou de um país ${ }^{28}$, como o caso do nosso estudo. Para as dimensões literacia comunicativa e crítica esses achados foram similares à validação do instrumento original HLS-14 em língua inglesa no Japão ${ }^{17}$ e na língua holandesa ${ }^{18}$, em que essas dimensões apresentaram consistência interna satisfatória. Porém, a literacia funcional no presente estudo ficou abaixo de 0,70 , fato que não ocorreu no Japão e na Holanda ${ }^{17,18}$, embora tenha apresentado valores substanciais. Também foi observado que apenas a LF (F1) apresentou problemas com a validade discriminante. Essa diferença encontrada na aplicação do instrumento na população brasileira em relação à japonesa e holandesa pode representar as características socioeconômicas da amostra em relação aos estudos anteriores, principalmente no quesito da educação. Estudos tem demonstrado que a educação formal no Brasil está atrasada em relação a países mais desenvolvidos. Este fato, por se tratar de um construto que avalia a literacia em saúde, pode apresentar essa interferência, os anos de escolaridade da educação formal brasileira não representam os mesmos de escolaridade da Holanda e Japão.

A amostra no presente estudo apresentou em sua maioria o ensino médio completo, que corresponde a onze anos de estudo, e mesmo assim no questionário aplicado em adultos de Piracicaba, as questões que apresentaram maior per- centual de baixa literacia em saúde foram desta dimensão LF, referente à dificuldade de ler bulas e compreender o significado de palavras, bem como a maioria concordou com a afirmação da dificuldade de compreensão do conteúdo das bulas de medicamentos.

$\mathrm{O}$ modelo inicialmente apresentou ajuste razoável de todos os índices: CFI $=0,886$, TLI $=0,86$, RMSEA $=0,085$ (IC90\%: 0,065-0,105), SRMR $=0,071$, qui-quadrado ( 74 graus de liberdade $=149,510, \mathrm{p}<0,001$. Os critérios usuais da AFC foram atendidos e são próximos aos encontrados na validação da versão original do construto no Japão ${ }^{17}$, e abaixo da versão holandesa ${ }^{18}$.

$\mathrm{Na}$ literacia funcional, foi encontrada uma relação entre a questão 4 e 5 que não foi detectada pelo modelo matemático, podendo apontar para uma variação na interpretação das questões por quem respondeu. Na versão original foi encontrada correlação entre as questões 3 e $5^{17}$. As questões são referentes a demora para ler, questão 4 , necessidade de ajuda para ler bulas e remédios respectivamente, questão 5 . A remoção da variável latente representada questão 5 , eleva os ajustes de forma considerável na análise [CFI $=0,930$, TLI $=0,912$, RMSEA $=0,075($ IC90\%: 0,046-0,093), SRMR $=0,064$, qui-quadrado $(64$ graus de liberdade $)=104,288, \mathrm{p}<0,001]$. A ajuda para ler pode trazer um confudimento que é o fato da pessoa precisar de ajuda para ler, não pelo aspecto de letramento, mas por questões fisiológicas de enxergar, mais do que compreender ou ter a capacidade de leitura, o que também pode ter interferido na validade discriminante. Na validação da versão holandesa houve relato desta confusão entre a ajudar para ler ou ajuda para compreender a leitura ${ }^{18}$. Essas questões devem ser revistas para uma próxima aplicação, pois na fase inicial de equivalência semântica não foram alteradas e adaptadas em relação ao questionário original. Abordar as dimensões da literacia que tem conceitos abstratos em um construto quantitativo é um desafio pela complexidade de avaliar a compreensão do indivíduo diante da informação em saúde, bem como sua habilidade de comunicação e julgamento nestas situações.

Com relação à simetria, não houve muita simetria, havendo maior concentração de respostas nos níveis mais altos da escala. A curtose sugere que as caudas são mais pesadas, com distribuição não normal. Na validação da versão, entretanto não ocorreu simetria ou curtose, exceto na dimensão funcional ${ }^{18}$.

No presente estudo não foram encontradas diferenças de literacia em saúde entre homens e 
Tabela 3. Distribuição da literacia em saúde para cada questão em adultos e idosos residentes em Piracicaba-SP, Brasil, 2015.

\begin{tabular}{|c|c|c|c|c|c|c|}
\hline Literacia & Questão & $\begin{array}{l}\text { Concordo } \\
\text { muito } \\
\text { n }(\%)\end{array}$ & $\begin{array}{l}\text { Concordo } \\
\text { n }(\%)\end{array}$ & $\begin{array}{c}\text { Nem } \\
\text { concordo, } \\
\text { nem } \\
\text { discordo } \\
\text { n }(\%) \\
\end{array}$ & $\begin{array}{l}\text { Discordo } \\
\text { n }(\%)\end{array}$ & $\begin{array}{l}\text { Discordo } \\
\text { muito } \\
\text { n (\%) }\end{array}$ \\
\hline \multirow[t]{5}{*}{ Funcional } & $\begin{array}{l}\text { Eu encontro palavras que não } \\
\text { consigo ler }\end{array}$ & $31(21,7)$ & $62(43,4)$ & $16(11,2)$ & $28(19,6)$ & $02(1,4)$ \\
\hline & $\begin{array}{l}\text { O tamanho da letra é muito } \\
\text { pequena para mim. }\end{array}$ & $32(22,4)$ & $66(46,2)$ & $11(7,7)$ & $29(20,3)$ & $01(0,7)$ \\
\hline & $\begin{array}{l}\text { O conteúdo é muito difícil de } \\
\text { entender. }\end{array}$ & $22(15,4)$ & $56(39,2)$ & $29(20,3)$ & $30(21)$ & $01(0,7)$ \\
\hline & $\begin{array}{l}\text { Demoro muito para ler (as } \\
\text { instruções). }\end{array}$ & $10(7)$ & $43(30,1)$ & $18(12,6)$ & $62(43,4)$ & $05(3,5)$ \\
\hline & $\begin{array}{l}\text { Eu preciso que alguém me ajude } \\
\text { a ler. }\end{array}$ & $07(4,9)$ & $20(14)$ & $16(11,2)$ & $70(49)$ & $26(18,2)$ \\
\hline \multirow[t]{5}{*}{ Comunicativa } & $\begin{array}{l}\text { Eu procuro informações em } \\
\text { vários lugares }\end{array}$ & $06(4,2)$ & $17(11,9)$ & $02(1,4)$ & $89(62,2)$ & $23(16,1)$ \\
\hline & $\begin{array}{l}\text { Eu encontro a informação que } \\
\text { preciso. }\end{array}$ & $05(3,5)$ & $19(13,3)$ & $11(7,7)$ & $92(64,3)$ & $10(7)$ \\
\hline & $\begin{array}{l}\text { Eu entendo a informação } \\
\text { encontrada. }\end{array}$ & $05(3,5)$ & $20(14)$ & $20(14)$ & $88(61,5)$ & $4(2,8)$ \\
\hline & $\begin{array}{l}\text { Eu falo minha opinião sobre a } \\
\text { doença ao meu médico, familiares } \\
\text { ou amigos }\end{array}$ & $04(2,8)$ & $33(23,1)$ & $8(5,6)$ & $80(55,9)$ & $13(9,1)$ \\
\hline & $\begin{array}{l}\text { Eu coloco em prática as } \\
\text { informações encontradas no meu } \\
\text { dia a dia. }\end{array}$ & $05(3,5)$ & $30(21)$ & $12(8,4)$ & $80(55,9)$ & $10(7)$ \\
\hline \multirow[t]{4}{*}{ Crítica } & $\begin{array}{l}\text { Eu sei quando as informações são } \\
\text { boas no meu caso. }\end{array}$ & $04(2,8)$ & $31(21,7$ & $23(16,1)$ & $72(50,3)$ & $08(5,6)$ \\
\hline & $\begin{array}{l}\text { Eu levo em conta se as } \\
\text { informações são verdadeiras }\end{array}$ & $03(2,1)$ & $25(17,5)$ & $19(13,3)$ & $79(55,2)$ & $12(8,4)$ \\
\hline & $\begin{array}{l}\text { Eu tenho conhecimento para julgar } \\
\text { se as informações são confiáveis. }\end{array}$ & $07(4,9)$ & $52(36,4)$ & $27(18,9)$ & $43(30,1)$ & $09(6,3)$ \\
\hline & $\begin{array}{l}\text { Eu pego informações que me } \\
\text { ajudam a tomar decisões de como } \\
\text { melhorar minha saúde. }\end{array}$ & $05(3,5)$ & $18(12,6)$ & $15(10,5)$ & $86(60,1)$ & $14(9,8)$ \\
\hline
\end{tabular}

Nota: Os dados não correspondem a 100\% devido a não resposta no questionário de literacia em saúde.

mulheres, o que não ocorreu na avaliação da versão validada do Japão onde os homens apresentaram mais altos escores de literacia que as mulheres de acordo com grupos etários, entretanto nos adultos mais velhos esta diferença estatística desaparece, sendo os escores menores que os mais jovens ${ }^{17}$. No presente estudo, os mais jovens apresentaram também maiores níveis de literacia.

Uma das limitações do presente estudo foi a etapa de avaliação por comitê de especialistas ter sido realizada por quatro pesquisadores da área da saúde, não sendo composta uma equipe multidisciplinar ou especialistas externos. Ressalta-se que o primeiro autor do questionário HLS-14 validado no Japão participou da avaliação após a retrotradução, aprovando a versão adaptada para lingua portuguesa brasileira obtida.

Apesar da amostra do presente estudo, fazer parte de um estudo longitudinal, a literacia em saúde medida pelo HLS-14 foi avaliada no recorte transversal, e não apresenta associação com sua dimensão temporal. Foi aplicado no baseline o questionário de Ishikawa et al. ${ }^{6}$, que foi a primeira versão da literacia comunicativa e crítica com cinco questões voltadas para a literacia em saúde bucal ${ }^{11}$. Nesta etapa, ainda não havia sido incorporada a literacia funcional. O questionário apresentou fácil aplicação e associação com 
fatores importantes sociais, de comportamento e clínicos de saúde bucal ${ }^{11}$. O grupo de pesquisa do Japão ${ }^{17}$ publicou em curto espaço de tempo um novo questionário, que além de poder se utilizado para diversas condições de saúde, apresentava modos de mensuração das três dimensões da literacia em saúde, e por isso, se tornou o instrumento de interesse para este estudo. Por ser um estudo inovador, torna-se necessário um aprofundamento para verificar o papel da literacia em saúde e condições de saúde, realizar os ajustes necessários para melhorar as propriedades psicométricas do estudo e realizar uma avaliação de critério para melhor compreensão do construto na população brasileira, intuito não presente no objetivo deste estudo. Os autores holandeses sugerem incorporar exemplos práticos nas questões para melhorar a compreensão das perguntas e consequentemente gerar respostas mais confiáveis ${ }^{18}$.

Conforme definido no modelo teórico conceitual de Macek et al. ${ }^{29}$, a literacia em saúde está associada à decisões e condições de saúde, e por isso, a grande relevância do tema para a comunidade científica e profissionais da saúde. Por esse motivo, ressaltamos que literacia em saúde

\section{Colaboradores}

ACP Marques participou no desenho do estudo, adaptações do instrumento, coleta dos dados piloto e redigiu o artigo científico. MF Silva Junior participou no desenho do estudo, adaptações do instrumento, coleta dos dados e redigiu o artigo científico. MJ Batista realizou o desenho do estudo, adaptações do instrumento, fez as análises estatísticas dos resultados e revisão final do artigo. MLR Sousa participou no desenho do estudo, adaptações do instrumento, realizou a supervisão de todas as fases do estudo e fez a revisão final do artigo. GP Alencar contribuiu na análise estatística, interpretação dos dados e revisão final do manuscrito. pode ser uma estratégia com impacto substancial sobre medidas preventivas e os resultados de saúde, contribuindo para reduzir as disparidades de saúde bucal e empoderando os indivíduos a tomar decisões adequadas, portanto, melhorando a qualidade de vida e reduzindo custos para o sistema de saúde ${ }^{30}$. Assim, a tradução, adaptação transcultural e avaliação psicométrica de um instrumento que mensure a literacia são passos essenciais para auxiliar em futuras investigações e tomadas de decisões em saúde.

\section{Conclusão}

O instrumento de literacia em saúde em Português (brasileiro) apresentou validade em uma população adulta e idosa brasileira, mas necessita de ajustes e nova avaliação de suas propriedades psicométricas para que o instrumento auxilie no planejamento de estratégias de promoção de saúde.

Futuros estudos que considerem a literacia em saúde como instrumento de tomada de decisões em saúde podem ser relevantes para saúde pública.

\section{Agradecimentos}

Ao financiamento pela Fundação de Amparo à Pesquisa do Estado de São Paulo (FAPESP) e Fundação de Amparo à Pesquisa do Estado de São Paulo/Coordenação de Aperfeiçoamento de Pessoal de Nível Superior (FAPESP/CAPES) e ao Espaço da Escrita pela tradução do artigo. 


\section{Referências}

1. Nutbeam D. Health promotion glossary. Health Promotion International. Geneva: Oxford University Press; 1998.

2. Rudd ER. Oral health literacy: correcting the mismatch. J Public Health Dent 2012; 72(Supl. 1):31-33.

3. Crondahl K, Karlsson LE. The Nexus Between Health Literacy and Empowerment: A Scoping Review. SAGE Open 2016; 6:1-7.

4. Nutbeam D. Health literacy as a public health goal: a challenge for contemporary health education and communication strategies into the 21st century. Health Promot Int 2000; 15(3):259-267.

5. Hibbard JH, Peters E, Dixon A, Tusler M. Consumer competencies and the use of comparative quality information - it is not just about literacy. Med Care Res Rev 2007; 64(4):379-394.

6. Ishikawa H, Takeushi T, Yano E. Measuring Functional, Communicative, and Critical Health Literacy among diabetic patients. Diabetes Care 2008; 31(5):874-879.

7. Atchison KA, Gironda MW, Messadi D, Der-Martirosian C. Screening for oral health literacy in an urban dental clinic. J Public Health Dent 2010; 70(4):269275.

8. Parker RM, Baker DW, Williams MV, Nurss JR. The Test of Functional Health Literacy in Adults (TOFLA): a new instrument for measuring patient's literacy skills. J Gen Intern Med 1995; 10(10):537-542.

9. Berkman ND, Sheridan SL, Donahue KE, Halpern DJ, Crotty K. Low Health Literacy and Health Outcomes: An Updated Systematic Review. Ann Intern Med 2011; 155(2):97-116

10. Ueno M, Takeuchi S, Oshiro A, Kawaguchi Y. Relationship between oral health literacy and oral health behaviors and clinical status in Japanese adults. J Dental Sci 2013; 8(2):170-176.

11. Batista MJ, Lawrence HP, Sousa MLR. Oral health literacy and oral health outcomes in an adult population in Brazil. BMC Public Health 2017; 18:60.

12. Nielsen-Bohlman L, Panzer AM, Kindig DA. Health Literacy: A prescription to End Confusion. Washington: National Academies Press; 2004.

13. Davis TC, Crouch MA, Long SW, Jackson RH, Bates P, George RB, Bairnsfather LE. Rapid assessment of literacy levels in adult primary care patients. Fam Med 1991; 23(6):433-436

14. Bass PFI, Wilson JF, Griffith CH. A shortened instrument for literacy screening. J Gen Intern Med 2003; 18(12):1036-1038.

15. Baker DW, Williams MV, Parker RM, Gazmararian JA, Nurss J. Development of a brief test to measure functional health literacy. Patient Educ Couns 1999; 38(1):33-42.

16. Lee JY, Rozier RG, Lee SY, Bender D, Ruiz RE. Development of a word recognition instrument to test health literacy in dentistry: The REALD-30--a brief communication. J Public Health Dent 2007; 67(2):9498.

17. Suka M, Odajima T, Kasai M, Igarashi A, Ishikawa $H$, Kusama M, Nakayama T, Sumitani M, Sugimori H. The 14-item health literacy scale for Japanese adults (HLS-14). Environ Health Prev Med 2013; 18(5):407415.
18. Van der Vaart R, Drossaert CH, Taal E, Klooster PM Hilderink-Koertshuis RT, Klaase JM, van de Laar MAF. Validation of the Dutch functional, communicative and critical health literacy scales. Patient Educ Couns 2012; 89(1):82-88.

19. Herdman M, Fox-Rushby J, Badia X. A model of equivalence in the cultural adaptation of HRQoL instruments: the universalist approach. Qual Life Res 1998; 7(4):323-335.

20. Reichenheim ME, Moraes CL. Operationalizing the cross-cultural adaptation of epidemological measurement instruments. Rev Saúde Pública 2007; 41(4):665-673.

21. Guillemin F, Bombardier C, Beaton D. Cross-Cultural adaptation of health-related quality of life measures: literature review and proposed guidelines. J Clin Epidemiol 1993; 46(12):1417-1432.

22. Silva-Junior MF, Sousa MLR, Batista MJ. Prospective cohort of adult oral health in Piracicaba, SP, Brazil. BMC Res Notes 2019; 12(1):221.

23. Kline P. The handbook of psychological testing. $2^{\text {a }}$ ed. London: Routledge; 2000.

24. Byrne BM. Structural Equation Modeling with Mplus: Basic Concepts, Applications, and Programming. London: Routledge; 2011.

25. $\mathrm{Hu} \mathrm{L}$, Bentler PM. Cutoff criteria for fit indices in covariance structure analysis: conventional criteria versus new alternatives. Struct Equ Modeling 1999; 6(1):1-55.

26. Fornell C, Larcker DF. Evaluating structural equation models with unobservable variables and measurement error. J Mark Res 1981; 18(1):39-50.

27. Cruvinel AFP, Méndez DAC, Oliveira JG, Gutierres E, Lotto M, Machado MAAM, Oliveira TM, Cruvinel T. The Brazilian version of the 20-item rapid estimate of adult literacy in medicine and dentistry. PeerJ 2017; 5:e3744.

28. Sorensen K, Van Den Broucke S, Fullam J, Doyle G, Pelikan J, Slonska Z. Health literacy and public health: a systematic review and integration of definitions and models. BMC Public Health 2012; 12:80.

29. Macek MD, Haynes D, Wells W, Bauer-Leffler S, Ann Cotten P, Parker RM. Measuring conceptual health knowledge in the context of oral health literacy: preliminary results. J Public Health Dent 2010; 70(3):197204.

30. Horowitz AM, Kleinman DV. Oral health literacy: a pathway to reducing oral health disparities in Maryland. J Public Health Dent 2012; 72(Supl. 1):26-30.
Artigo apresentado em 27/08/2018

Aprovado em 26/11/2018

Versão final apresentada em 28/11/2018 
